\section{Ionisation by Positive Ions}

IN recent years many theories of discharges in gases have been proposed which do not afford a consistent explanation of the principal properties of currents obtained in gases under various conditions. According to a theory which has been much advocated, ${ }^{1}$ it is said that atoms of the gas are not ionised by single impacts of electrons in discharges at pressures greater than that corresponding to the minimum sparking potential, since the electrons lose their energies in producing metastable atoms and therefore cannot attain the ionising potential. So far as the action of electrons is concerned, it has already been shown that this theory of collisions is inconsistent with the ordinary properties of glow discharges. ${ }^{2}$

Recent theories ${ }^{3}$ also involve unsatisfactory hypotheses with regard to the action of positive ions in contributing to the conductivity.

In these theories it is maintained that atoms or molecules of a gas cannot be ionised by the collisions of positive ions unless the kinetic energy of the ions is of several thousand volts. Thus if the ionising potential of an atom be 10 volts, a hydrogen ion would require an energy of at least 4500 volts in order to ionise the atom. ${ }^{4}$ It has therefore been concluded that in discharges where the potentials between the electrodes are less than a thousand volts, the action of the positive ions in contributing to the conductivity is limited to the liberation of electrons from the negative electrode, and in order to explain the corona discharge it was. found necessary to assume that the emission of electrons from the electrode is independent of the energy of the positive ions acquired by moving under the electric force in the gas. The objections to these hypotheses as a basis of a general theory of discharges have been pointed out. ${ }^{5}$

The modern theories thus differ from the wellknown theory of the disruptive discharge which was deduced from experiments made some years ago in the Electrical Laboratory, Oxford.

In this theory, in order to reconcile the results of the experiments on currents between parallel-plate electrodes with experiments on the corona discharges, it is assumed that molecules or atoms of the gas are ionised by the collisions of positive ions in discharges maintained by potentials of a few hundred volts, where a small proportion of the ions acquire energies of about twenty volts. When the electric force is small and the gas pressure is large, the rate of ionisation due to positive ions is very small, but it is increased when the force is increased or the pressure reduced. Thus the ionisation increases with the kinetic energy acquired by the positive ions in moving under the action of the electric force.

We have recently made direct experiments to determine the effects of positive ions in hydrogen, the apparatus being designed so that it was possible to separate the effects of the liberation of electrons from the negative electrode from the effect due to the ionisation of atoms of the gas. We find that the number of electrons generated by positive ions in a current between parallel plates depends on the electric force and on the pressure of the gas. There is a large increase in the number of electrons thus generated, corresponding to a small increase in the force with forces of the order of 100 volts per centimetre and pressures of the order of half a millimetre. Also with copper plates $2 \mathrm{~cm}$. apart the number of electrons liberated from the negative electrode is small compared with the number of molecules of the gas that are ionised by the impacts of the positive ions.

These experiments show that the energies of the positive ions in the collisions in which molecules are ionised are of the same order as that of the electrons,

so that the velocities of the positive ions in these collisions are of the order of one-hundredth of the velocities of the electrons.

These results therefore confirm the earlier theory of disruptive discharge, given in the treatise "Electricity in Gases".

xford, Aug. 18.

J. S. TOWNSEND.

F. Llewellyn Jones.

1 T. R. Merton and J. G. Pilley, Proc. Roy. Soc., A, 107, 411; 1925. R. d'E. Atkinson, Proc. Roy. Soc., A, 119, $335 ; 1928$.

Phil Mag, 5, 695; 1928. Proc. Roy. Soc., A, 124, 533 ; 1929.

J. Taylor, Proc. Roy. Soc., A, 14, 73; 1927. K. Zuber, Ann. Phys. (12), 6, 665; 1932 .

Sir J. J. Thomson, Phil. Mag., 48, 1, July 1924

5 J. S. Townsend, Phil. Mag., 45, 444, March 1923. L. G. H. Huxley, Phil. Mag., 3, 1056, and 4, $899 ; 1927$.

\section{Polarisation of Echoes from the Heaviside Layer}

ONE of the most fruitful methods of investigating the Heaviside layer is that originated by Breit and Tuve, in which a series of short impulses of the order of 0.2 of a millisecond in duration are transmitted and the direct ray and echoes reflected at nearly normal incidence are observed at a neighbouring station. The echo patterns observed are often very complex and it has been found that a single $F$ layer echo, say, is often split into two fairly closely spaced components. This has been attributed by Appleton to the double refraction suffered by a ray in passing through the ionised regions of the upper layer.

The double refraction is caused by the earth's magnetic field. According to the theory, these two components should be oppositely circularly or elliptically polarised. A complete proof that the splitting of echoes is due to double refraction should involve a demonstration of this fact. A recent experiment, in which this result is clearly demonstrated, con. stitutes a striking proof of the magneto-ionic theory of radio transmission.

For the purpose of determining the state of polarisation of the downcoming waves, two separate vertical tuned loop aerials perpendicular to each other, as in a Bellini-Tosi direction finder, were used at the receiving end. These were coupled in the usual way to a search coil which fed the receiver. The output of this actuated a cathode ray oscillograph in the normal way for demonstrating the echoes. The object is to use the double frame as a polarimeter. This can be achieved as follows: The aerial is set so that the angle between the two frames is bisected by the incoming direct or surface ray. The aerials are then tuned so that the currents in the two are in phase with the e.m.f.'s, which are also in phase. The search coil of the goniometer can then be set at $45^{\circ}$ so as to receive no signal. The aerials are then mistuned, one being increased in natural period and the other reduced. When the e.m.f.'s in the aerials are in phase, the resultant is a rotating field in the goniometer and no position of balance can be found.

Consider a ray returned from the Kennelly-Heaviside layer : if this is circularly polarised, then the e.m.f.'s in the two frames will be $90^{\circ}$ out of phase. If the mistuning of the aerials has been properly carried out so that the current in one is advanced $45^{\circ}$ on the e.m.f. and retarded $45^{\circ}$ in the other, then, when the e.m.f.'s are $90^{\circ}$ out of phase, the currents will either be in phase or $180^{\circ}$ out of phase, according to the direction of circular polarisation. It follows that for a circularly polarised wave a balance can be obtained with the pointer of the goniometer either at $+45^{\circ}$ or $-45^{\circ}$, according to the direction of circular polarisation.

Let $A$ and $B$ in Fig. 1 represent the components of

No. 3280, Vor. 130] 